\title{
Coagulation in a Continuously Stirred Tank Reactor
}

\author{
E. M. HENDRIKS AND R. M. ZIFF
}

Institut für Theoretische Physik, Universität zu Köln, Zülpicher Str. 77, D-5000 Köln 41, West Germany, and Department of Chemical Engineering, University of Michigan, Ann Arbor, Michigan 48109

Received July 30, 1984; accepted October 25, 1984

\begin{abstract}
Smoluchowski's coagulation equation with coagulation rates $K_{i j} \propto(i j)^{\omega}$ describing the temporal evaluation of the size distribution $c_{k}$ (concentration of $k$-mers) is studied in the presence of a source term $a \delta_{k 1}$ and a sink term $-b c_{k}$. With $a^{\prime}=a / b^{2}$ one finds that a steady state is reached, exhibiting gelation when $\omega>\frac{1}{2}$ and $a^{\prime}>a_{0}(\omega)$, where $a_{0}(\omega)$ depends on $\omega\left(a_{0}(\omega)=\infty\right.$ for $\omega<\frac{1}{2}, a_{0}(1)=\frac{1}{4}, a_{0}(\omega)$ $=0$ for $\omega>1$ ). For $a^{\prime}<a_{0}(\omega)$ the asymptotic behavior of the $c_{k}$ is dominated by exponential decrease, for $a^{\prime}>a_{0}(\omega)$ it has an algebraic tail. The case $\omega=1$ is singular in that also nongelling steady states have an algebraic tail $\propto k^{\beta}$, where $\beta$ depends on $a^{\prime}$. 1985 Academic Press, Inc.
\end{abstract}

\section{INTRODUCTION}

In this paper we study properties of the solutions, in particular steady-state solutions, of the population balance equation

$$
\begin{aligned}
\dot{c}_{\mathrm{k}}=\frac{1}{2} \sum_{i+j=k} K_{i j} c_{i} c_{j}-c_{k} \sum_{j=1}^{\infty} & K_{k j} c_{j} \\
& +a_{k}-b_{k} c_{k}
\end{aligned}
$$

describing coagulation of particles in a continuously stirred tank reactor (1-3). The coagulation rates are represented by the numbers $K_{i j} ; c_{k}(t)$ is the concentration of particles containing $k$ structural units ( $k$-mers), $a_{k}$ is the source term, and $b_{k} c_{k}$ is the removal term. We shall always take $a_{k}=a \delta_{k 1}$ (monomer source) and $b_{k}$ as a constant; then $a$ is the rate at which monomers are being fed into the reactor, and $1 / b$ is the mean residence time. An important quantity, associated with the distribution $c_{k}(t)$, is its first moment, or the total mass of all the finite size particles:

$$
M_{1}(t)=\sum_{k=1}^{\infty} k c_{k}(t) .
$$

A balance equation for this quantity can be formally derived from [A] (with $a_{k}=a \delta_{k 1}$ and $b_{k}=b$ ) but this derivation is only valid when the $c_{k}$ decrease rapidly enough as a function of $k$. In general, only the inequality

$$
\dot{M}_{1} \leqslant a-b M_{1}
$$

can be derived by multiplying $[\mathrm{A}]$ on both sides by $k$ and summing over all $k$. The above-mentioned balance equation has the equal sign. In some cases, depending on the coagulation rates $K_{i j}$, solutions exist for which the strict inequality sign holds. It seems that these solutions do not conserve mass, as the RHS of $[\mathrm{C}]$ represents the difference of inand outflowing mass. The deficit is caused by the formation of an infinite cluster, the mass of which is not present in [B]. This phenomenon is called gelation, and has been discussed in (1, 2, 4-9). In the absence of source and removal terms, criteria whether or not gelation occurs have been developed and in some cases bounds on the gelation time have been derived. For example, when $K_{i j} \sim(i j)^{\omega}$ for large $i$ and $j$, gelation occurs only when $\omega>\frac{1}{2}$ and at the critical point the $c_{k}$ decay as $k^{-\tau}$ with a critical exponent $\tau$ $=\omega+\frac{3}{2}(4,7)$. The same is true of the steadystate solution when only a source is present (1). The amplitude of this algebraic tail is in both cases proportional to the square root of the rate of gel formation. White (1) has given an existence proof for a class of kernels, source, and removal terms, which was later generalized by Crump and Seinfeld (2). These authors' results may be formulated as follows. 
Assume a monomer source $a_{k}=a \delta_{k 1}$ and kernel and removel terms satisfying the relations: $K_{i j}<$ const. $(i j)^{\omega+\beta}$ and $b_{k} \geqslant$ const. $k^{\beta}$, where $\beta \geqslant 0$. Then, if $\omega<1$ a nonnegative steady-state solution to Eq. [A] exists, having finite mass. If the stronger condition $\omega<\frac{1}{2}$ is satisfied, this solution is mass-conserving (nongelling) and has finite moments of all order. As an example (2) one may apply this to aerosols undergoing Brownian coagulation $\left(K_{i j} \leqslant\right.$ const. $\left.(i j)^{1 / 3}\right)$ and gravitational sedimentation $\left(b_{k} \geqslant\right.$ const. $\left.k^{2 / 3}\right)$. In this case, $\omega$ $=-\frac{1}{3}$ and hence gravitational sedimentation acts as a sink, strong enough to preclude gelation phenomena in aerosol systems.

In the same paper it has been conjectured that $\omega=\frac{1}{2}$ is actually the largest possible exponent for which mass-conserving solutions exist. This is not true, however, as we shall show that it is possible to construct examples for which the removal term is weak enough to imply $\omega>\frac{1}{2}$ and still strong enough to preclude gelation.

In this paper we restrict ourselves to separable kernels of the form $K_{i j}=s_{i} s_{j}$. We prove, in the Appendix, existence and unicity of the steady-state solution irrespective of the functional form of $s_{k}$, using an idea of Leyvraz (9). This covers many cases in which the conditions under which the theorems in (2) are derived are not satisfied and thus the class of kernels for which existence of steadystate solutions is known is enlarged. For $s_{k}$ $=k$ we investigate for which values of $\omega, a$ and $b$ gelation occurs and what the asymptotic properties of the distribution are for gelling and nongelling steady states. These results are expected not to change when $K_{i j}$ is not strictly equal to $(i j)^{\omega}$, but only asymptotically for large $i$ and $j$. For $\omega=0$ we give the complete time-dependent solution, which is a generalization of a result of Lushnikov and Piskunov (3).

\section{BASIC CONCEPTS}

\subsection{Basic Equation}

The coagulation process in a continuously stirred tank reactor is described by the set of differential equations

$$
\begin{aligned}
\dot{c}_{k}=\frac{1}{2} \sum_{i+j=k} K_{i j} c_{i} c_{j}-c_{k} \sum_{j=1}^{\infty} K_{k j} c_{j} & \\
& +a \delta_{k 1}-b c_{k} .
\end{aligned}
$$

For a given initial state, these equations determine the time evolution of the size distribution, $c_{k}(t)$. The rate at which $i+j$ clusters are formed out of $i$ - and $j$-clusters is taken to be $K_{i j} c_{i} c_{j}$. Monomers are fed into the system at a rate $a$ and mass is removed from the system at a rate proportional to the amount present. In this paper we shall almost exclusively study the process for initial conditions $c_{i}(0)=0$, and take the kernel $K_{i j}$ of the form $s_{i} s_{j}$ where $s_{k}$ is a measure for the effective, reactivity of a $k$-mer.

Changing to new variables $t^{\prime}=b t$ and $c_{k}^{\prime}$ $=c_{k} / b$ such that $c_{k}^{\prime}\left(t^{\prime}\right)=c_{k}(t)$ we obtain an equation of exactly the same form as [1], in which $b$ is replaced by unity and $a$ by $a / b^{2}$ $=a^{\prime}$. Omitting the primes we write

$$
\begin{aligned}
\dot{c}_{k}=\frac{1}{2} \sum_{i+j=k} s_{i} s_{j} c_{i} c_{j}-c_{k} s_{k} \sum_{j=1}^{\infty} s_{j} c_{j} & \\
& +a \delta_{k 1}-c_{k} .
\end{aligned}
$$

To obtain a solution of the original equation from a solution of this equation, one simply has to make the substitutions $c_{k} \rightarrow b c_{k}, t \rightarrow$ $b t$, and $a \rightarrow a / b^{2}$.

In general, Eq. [2] resists solution, but often certain properties of the distribution can be obtained. Of interest are the values of the lower moments $M_{n}=\sum k^{n} c_{k}$ and the asymptotic behavior of $c_{k}$ for large $k$. We shall be mainly interested in steady-state solutions and their properties. These are solutions for which $\dot{c}_{k}$ is zero. In the Appendix we give a rather technical proof of the existence of such solutions.

\subsection{Mass Balance Equation}

In the new units, the rate at which mass is flowing into the system is $a$. The amount of mass leaving the system per unit time is given by the value of the first moment of the size distribution:

$$
M_{1}(t)=\sum_{k=1}^{\infty} k c_{k}(t) .
$$


From [2], by multiplying by $k$ and summing over all $k$, the following equation can be derived:

$$
\dot{M}_{1}=a-M_{1}-\lim _{L \rightarrow \infty} \sum_{i=1}^{L} \sum_{j=L-i+1}^{\infty} i s_{i} s_{j} c_{i} c_{j} .
$$

If the last term of the RHS is zero, this becomes the mass balance equation, which expresses that the increase of mass in the system is a result of a difference between inflow, $a$, and outflow $M_{1}$. This is the case whenever

$$
\lambda=\sum_{i=1}^{\infty} k s_{k} c_{k}
$$

is finite. This quantity may diverge, however, so in general we only have

$$
\dot{M}_{1} \leqslant a-M_{1} \text {. }
$$

The loss of mass is due to the formation of an infinite cluster, the mass of which is not contained in $M_{1}$ (which is a sum over all finite clusters). This phenomenon is called gelation (4-9). We shall call solutions for which $\dot{M}_{1}(t)$ becomes strictly less than $a$ $-M_{1}$ at some point: gelling states as opposed to nongelling states.

\subsection{Moment Equations}

An important mathematical tool when investigating properties of the solutions of Eq. [2] is furnished by the moment equations. The moments of the distribution are defined in the usual way:

$$
M_{n}(t)=\sum_{k=1}^{\infty} k^{n} c_{k}(t) .
$$

If we multiply [2] by $k^{n}$, sum, and rearrange terms we find

$$
\dot{M}_{n}=a-M_{n}+\frac{1}{2} \sum_{k=1}^{n-1}\left(\begin{array}{l}
n \\
k
\end{array}\right) S_{k} S_{n-k},
$$

where

$$
S_{n}(t)=\sum_{k=0}^{\infty} k^{n} s_{k} c_{k}(t) .
$$

In deriving Eq. [8] we have omitted a term like the last term in Eq. [4] which vanishes only when $S_{n}(t)$ is finite $(2,4,7)$. Provided this, the moment Eqs. [8] are valid. It is possible that $S_{n}(t)$ is infinite for $n>N$ and finite for $n \leqslant N$. In that case the moment equations are valid only up to $n=N$. For gelling states $N=0$. Only for special choices of $s_{k}$, such as $s_{k}=1$ or $s_{k}=k$ are the moment equations closed.

\subsection{Generating Functions}

Another important mathematical tool is the (moment) generating functions. We define two of them as

$$
\begin{aligned}
& f(x, t)=\sum_{k=1}^{\infty} s_{k} c_{k}(t) e^{k x} \\
& g(x, t)=\sum_{k=1}^{\infty} c_{k}(t) e^{k x}
\end{aligned}
$$

for complex $x$. The series converge for $\operatorname{Re}(x)$ $<0$ when $c_{k}(t)$ is a solution of [2], since in order for the solution to make sense both $S_{0}$ $=\sum s_{k} c_{k}(t)$ and $M_{0}=\sum c_{k}(t)$ must converge. The function $g$ generates the moments about $x=0$ (as far as they exist) and the cluster size distribution about $x=-\infty$. We have the following explicit formulas

$$
M_{n}(t)=(2 \pi i)^{-1} n ! \int g(x, t) x^{-n-1} d x
$$

and

$$
c_{k}(t)=(2 \pi i)^{-1} \int g(\log z, t) z^{-k-1} d z,
$$

where the integration paths are closed contours around the origin in the respective complex planes. Multiplying Eq. [2] by $e^{k x}$ and summing over all $k$, we find that $f$ and $g$ are related by the equation

$$
\partial g / \partial t=f^{2} / 2-f S_{0}+a e^{x}-g,
$$

where $S_{0}=f(0, t)=\Sigma s_{k} c_{k}$. In the case of a gelling state we can obtain the asymptotic (large $k$ ) behavior of the size distribution from [14]. Since $M_{1}(t)$ is finite we may expand $g$ about $x=0$ as

$g(x, t)=M_{0}+M_{1} x+\mathrm{O}\left(x^{2}\right) \quad(x \uparrow 0)$. 
We rewrite [14], using $S_{0}^{2}=2\left(a-M_{0}-\dot{M}_{0}\right)$ (which follows from [14] by putting $x=0$ ) as

$$
\begin{aligned}
f=S_{0}-\left[2 \left(a-M_{0}\right.\right. & -\dot{M}_{0} \\
& \left.\left.-a e^{x}+g+\dot{g}\right)\right]^{1 / 2} .
\end{aligned}
$$

Expanding this about $x=0$, using [15], yields in lowest order:

$$
\begin{gathered}
f \cong S_{0}-\left[2\left(a-M_{1}-\dot{M}_{1}\right)\right]^{1 / 2}(-x)^{1 / 2} \\
(x \uparrow 0) .
\end{gathered}
$$

This corresponds, from the definition [10], to the asymptotic large- $k$ behavior of the size distribution

$$
\begin{gathered}
s_{k} c_{k} \sim\left[\left(a-M_{1}-\dot{M}_{1}\right) / 2 \pi\right]^{1 / 2} k^{-3 / 2} \\
(k \rightarrow \infty) .
\end{gathered}
$$

The coefficient is thus expressed in terms of $M_{1}$, which is an unknown function of $t$. For nongelling states this coefficient vanishes, because for these states the mass balance equation $\dot{M}_{1}=a-M_{1}$ holds. We may interpret the quantity $\dot{g} \equiv a-\dot{M}_{1}-M_{1}$ as the rate of gel formation. Equation [18] is the generalization of a similar formula derived in (7) to the case in which a source term and a removal term is present. In the steady state, $\dot{M}_{1}=0$.

In general we expect the size distribution to have the asymptotic behavior

$$
c_{k} \sim A \eta^{k} k^{\beta} \quad(k \rightarrow \infty),
$$

where $A, \eta$, and $\beta$ are parameters depending in principal on time and on the source rate $a$. The parameter $\eta$ is related to the radius of convergence of $f$ and $g$, and becomes unity for gelling states. The exponent $\beta$ does not depend on time and is related to the nature of the singularity of $g$ at the radius of convergence.

\section{COAGULATION RATES $K_{i j}=(i j)^{\omega}$}

In the following we restrict ourselves to coagulation kernels of the form $K_{i j}=(i j)^{\omega}$ $\left(s_{k}=k^{\omega}\right)$. These have been studied in $(4,7)$. For different choices of $\omega$ various coagulation mechanisms are described by this form (2) in the limit of large clusters. It has been shown that in the absence of source and removal terms, and for a large class of initial conditions, gelation occurs for $\omega>\frac{1}{2}$ and is absent for $\omega<\frac{1}{2}(4,7)$ and some bounds on the gelation time have been derived. We shall investigate the nature of the solution and in particular of the steady state for various choices of $\omega$. The existence of a unique steady state will be proved in the Appendix. Beside the question of asymptotic behavior of the $c_{k}$ it is of interest to know the answer to the questions: For which value of $a$ and $\omega$ does the system reach a gelling steady state? What is the dependence of the rate of gel formation $\left(a-M_{1}\right)$ on $a$ ? We start with the simpler case $\omega=0\left(K_{i j}=1\right)$ for which the time-dependent problem was solved in (3) with a source but no removal term. It is an easy matter to generalize the result to include removal.

\subsection{The Case $\omega=0\left(K_{i j}=1\right)$}

In the case of constant coagulation rates, as in Smulochowski's original coagulation equation (10), the generating functions $f$ and $g$, defined in [10] and [11], are equal $\left(s_{k}=k^{\omega}\right.$ $=1$ ), so that [14] becomes a closed nonlinear partial differential equation for $g$. Here it is more convenient to use another generating function, $h$, defined by

$$
\begin{aligned}
h(x, t) & =\sum_{k=1}^{\infty} c_{k}(t)\left(e^{k x}-1\right) \\
& =g(x, t)-M_{0} .
\end{aligned}
$$

If we substract from [14] the same equation with $x=0$, we find that $h$ satisfies

$$
\partial h / \partial t=h^{2} / 2-h+a\left(e^{x}-1\right)
$$

which we want to solve with the initial condition $h(x, 0)=0$. It is not difficult to generalize this to arbitrary initial conditions. The steady state, however, does not depend upon $c_{k}(0)$. Abbreviating $\psi=a\left(e^{x}-1\right)$ we can treat equation [21] as just an ordinary differential equation, in which $\psi$ occurs as a parameter, and solve it by separating variables, with the result: 
$h(x, t)$

$$
=\frac{2 \psi \tanh \left[(t / 2)(1-2 \psi)^{1 / 2}\right]}{(1-2 \psi)^{1 / 2}+\tanh \left[(t / 2)(1-2 \psi)^{1 / 2}\right]} .
$$

The steady state is obtained from this by substituting $t=\infty$. It corresponds to a zero of the RHS of Eq. [21]:

$$
h(x, \infty)=1-(1-2 \psi)^{1 / 2}
$$

agreeing with a result of White (1). A corresponding expression in (3) for the generating function of the distribution, valid when there is no removal, can be obtained from [22] by changing back to the original variables $(h \rightarrow$ $\left.b h, t \rightarrow b t, a \rightarrow a / b^{2}\right)$ and letting $b \rightarrow 0$. For finite times the expression [22] represents a meromorphic function with an infinite number of simple poles on the real axis to the right of $x_{0}=\log (1+1 / 2 a)$. An asymptotic formula for these poles is

$$
\begin{gathered}
x_{n} \cong \log \left[1+(1 / 2 a)+(2 / a)(\pi n / t)^{2}\right] \\
(n=1,2, \cdots) \quad(t \rightarrow \infty) .
\end{gathered}
$$

It follows from [13], [20], and [22] that the exact distribution can be written in the form of a series (for $t$ finite)

$$
\begin{aligned}
c_{k}(t)=(2 / a t) \sum_{n=1}^{\infty}[1 & \left.-2 a\left(x_{n}-1\right)\right] \\
& \times \exp \left[-(k+1) x_{n}\right] .
\end{aligned}
$$

So that the large- $k$ behavior is dominated by a purely exponential tail distribution. For $t \rightarrow \infty$, however, the poles become dense on the real axis to the right of $x_{0}$. For $t=\infty$ the size distribution can be calculated in explicit form from [23]:

$$
c_{k}(\infty)=\left|\left(\begin{array}{c}
1 / 2 \\
k
\end{array}\right)\right|(1+2 a)^{1 / 2}\left(\frac{2 a}{1+2 a}\right)^{k}
$$

which has the asymptotic behavior

$$
\begin{gathered}
c_{k}(\infty) \sim \frac{1}{2}\left(\frac{1+2 a}{\pi}\right)^{1 / 2} k^{-3 / 2}\left(\frac{2 a}{1+2 a}\right)^{k} \\
(k \rightarrow \infty),
\end{gathered}
$$

i.e., exponential decay dressed with an algebraic factor. The value of the first moment
$M_{1}(t)$, which is the amount of mass flowing out of the system, can be found from [22] by differentiating with respect to $x$ :

$$
M_{1}(t)=(\partial h / \partial x)_{x=0}=a\left(1-e^{-t}\right)
$$

which is the solution of the mass balance equation $\dot{M}_{1}=a-M_{1}$ with $M_{1}(0)=0$. Therefore this model does not suffer a gelation transition. In the steady state, $M_{1}=a$.

So we have shown that the model with $\omega$ $=0$ can be solved explicitly, and the timedependent solution leads to a steady state with mass conserved and therefore does not undergo a gelation transition.

\subsection{The Case $\omega=1\left(K_{i j}=i j\right)$}

For $\omega=1$, Eq. [14] becomes a nonlinear partial differential equation for $g(x, t)$ (since $f=\partial g / \partial x$ in this case) which resists solution. The second moment $M_{2}(t)$, however, satisfies a closed equation:

$$
\dot{M}_{2}=M_{2}^{2}-M_{2}+a .
$$

As follows from [8], it is valid provided $S_{2}(t)$ $=M_{3}(t)<\infty$. The solution for $M_{2}(0)=0$ can be written as

$$
M_{2}(t)=\frac{2 a \tanh \left[(t / 2)(1-4 a)^{1 / 2}\right]}{(1-4 a)^{1 / 2}+\tanh \left[(t / 2)(1-4 a)^{1 / 2}\right]} .
$$

There are two qualitatively different cases. If $a<\frac{1}{4}, M_{2}(t)$ remains bounded for all times and approaches the steady-state value

$$
M_{2}(\infty)=\left[1-(1-4 a)^{1 / 2}\right] / 2 \text {. }
$$

This implies that the mass balance equation ([8] for $n=1$ ) is valid, so the system never gels and approaches a nongelling steady state with $M_{1}(\infty)=a$. If $a>\frac{1}{4}$, however, $M_{2}(t)$ diverges within a finite amount of time. The point $t_{\mathrm{c}}$ at which $M_{2}(t)$ becomes infinite, the gel point, corresponds to a zero of the dominator in [30]:

$$
\begin{aligned}
t_{\mathrm{c}}=2(4 a-1)^{-1 / 2} & {[\pi} \\
& \left.-\arctan (4 a-1)^{1 / 2}\right] .
\end{aligned}
$$

So for $a>\frac{1}{4}$ the system approaches a gelling steady state, with $M_{1}(\infty)<a$. The asymptotic behavior of the corresponding size distribu- 
tion follows from [18], with $\dot{M}_{1}=0$ and $s_{k}$ $=k$, as

$$
\begin{gathered}
c_{k} \sim\left[\left(a-M_{1}\right) / 2 \pi\right]^{1 / 2} k^{-5 / 2} \\
(k \rightarrow \infty)
\end{gathered}
$$

Next we investigate the asymptotic behavior for $a<\frac{1}{4}$.

First we note that the moment equations [8] in the steady state can be written here as

$$
\begin{gathered}
M_{n}=\left(1-n M_{2}\right)^{-1}\left\{\frac{1}{2} \sum_{k=2}^{n-2} M_{k+1} M_{n-k+1}+a\right\} \\
n=3,4, \ldots,
\end{gathered}
$$

where $M_{2}$ is given by [31]. Using this all moments can be calculated recursively. However, it seems to follow from [34] that when $n>\left(1 / M_{2}\right)$ the moments become negative, i.e., unphysical. In fact, the moments with $n$ $>\left(1 / M_{2}\right)$ must be infinite. This follows, for instance, from the solution of the time-dependent moment equations (which can also be solved recursively in principle). This behavior indicates that for $a<\frac{1}{4}$ there is still an algebraic tail distribution, although the steady state is a nongelling one. To find the exponent we use the equation for the generating function in the steady state which follows from [14]:

$$
f^{2} / 2-f M_{1}+a e^{x}-g=0,
$$

where $f=f(x, \infty)=g^{\prime}(x, \infty)$ and $M_{1}=f(0$, $\infty)$. We assume that there is an (integer) value $N$ of $n$ such that $M_{n}$ is finite for $n \leqslant N$ and infinite for all $n>N$ because of an algebraic tail in $c_{k} \sim k^{-\beta}$ which leads to the singularity $\sim(-x)^{\beta-1}$ in the generating function. This means that about $x=0$ the generating functions $f$ and $g$ will have the expansion

$$
\begin{gathered}
g \cong \sum_{n=0}^{N} M_{n} x^{n} / n !+\gamma(-x)^{\delta} \\
(N<\delta<N+1)
\end{gathered}
$$

and

$$
f=g^{\prime} \cong \sum_{n=0}^{N-1} M_{n+1} x^{n} / n !-\gamma \delta(-x)^{\delta-1} .
$$

We substitute these into Eq. [35] and equate the coefficient of the various powers $x^{n}$ to zero. For $n \leqslant N$ we recover the moment Eqs. [34]. The term proportional to $(-x)^{\delta}$ leads to

$$
\delta=1 / M_{2}
$$

and $\gamma$ remains undetermined. Hence $g$ has a singularity $\gamma(-x)^{1 / M_{2}}$ in $x=0$ to which corresponds an algebraic tail in the size distribution

$$
c_{k} \sim A(a) k^{-1-1 / M_{2}},
$$

where $M_{2}$ is given as a function of $a$ by Eq. [31]. The coefficient $A(a)$ remains undetermined. If we increase the source rate $a, M_{n}$ becomes infinite at the value $a_{n}=(n-1)$ / $n^{2}$. For $a>a_{2}=\frac{1}{4}$ the second moment itself is infinite and the system gels. In this range $M_{1}(a)$ becomes strictly less than $a$. We note that Eq. [29] can be solved for general initial condition $M_{2}(0)$, allowing some mass to be present in the reactor at $t=0$. One finds that $M_{2}(t)$ diverges when $M_{2}(0)>\frac{1}{2} \mid 1+(1$ $-4 a)^{1 / 2}$, even when $a<\frac{1}{4}$, in a finite time $t_{\mathrm{c}}$. However, the steady state, which is unique, depends solely on $a$ and not on $M_{2}(0)$ and therefore should be nongelling. This seems in contradiction with the fact that here $M_{2}(t)$ $=\infty$ for all (finite) $t>t_{\mathrm{c}}$. The solution to this paradox lies in the fact that the approach to the steady state is nonuniform in $k$, so that it is possible that $M_{2}(t)=\infty$ for all $t$ $>t_{\mathrm{c}}$ and yet $M_{2}(\infty)=$ finite. At all finite times the distribution has an algebraic tail as in [10] causing $M_{2}$ to diverge, the amplitude of which vanishes as $t \rightarrow \infty$.

\subsection{General $\omega\left(K_{i j}=(i j)^{\omega}\right)$}

For arbitrary $\omega(\neq 1, \neq 0)$, none of the higher moments can be found in explicit form. The moment equations

$$
\dot{M}_{n}=a-M_{n}+\frac{1}{2} \sum_{k=1}^{n-1}\left(\begin{array}{l}
n \\
k
\end{array}\right) M_{k+\omega} M_{n-k+\omega}[40]
$$

are not closed. However, for $\omega<1$ the equation with $n=2$ :

$$
\dot{M}_{2}=a-M_{2}+M_{1+\omega}^{2}
$$


implies, since $M_{1+\omega}<M_{2}$, that the second moment is bounded by the solution of Eq. [29] (which was strictly valid for $\omega=1$ ), i.e., by the expression [30].

Since $M_{1+\omega}<M_{2}$ and gelation occurs only when $M_{1+\omega}=\infty$, it follows at least for $a$ $<\frac{1}{4}$ the system never gels. In the absence of removal it is known that the system suffers gelation for $\omega>\frac{1}{2}(1)$. Therefore we expect that there is a number $a_{0}(\omega)$ such that gelation occurs for $a>a_{0}(\omega)$ and does not for $a$ $<a_{0}(\omega)$. In the case of gelation, the size distribution asymptotically behaves as $c_{k}$ $\sim k^{-(3 / 2+\omega)}$ (see [18]). On the other hand $M_{1}$ has to be finite, i.e., $k c_{k}$ has to converge. This is only consistently possible when $\omega>\frac{1}{2}$. Hence gelation is precluded for $\omega<\frac{1}{2}$, so that $a_{0}(\omega)=\infty$ for $\omega<\frac{1}{2}$. It follows from the results of Section 3 that $a_{0}(1)=\frac{1}{4}$.

To find the asymptotic behavior of the size distribution for $a<a_{0}(\omega)$ we may proceed as before, using Eq. [36]. In this case $f$ is not simply the derivative of $g$, but it follows from the definitions [10] that when $g$ has a singular term $\propto(-x)^{\delta}$ about $x=0$ then $f$ has a corresponding singular term $(-x)^{\delta-\omega}$. If we substitute this into [35] we find that it is impossible to match the coefficients of the singular term since the powers are different. We conclude that $g$ cannot have a singularity in $x=0$ for $a<a_{0}(\omega)$, so that $c_{k}$ must have an asymptotic behavior as in Eq. [19], with $\eta<1$. This means that $f(x)$ and $g(x)$ both have a singularity in $x=x_{0}=-\ln (\eta)$. Consistent with [35] one finds $f \propto\left(x_{0}-x\right)^{1 / 2}$ for $x \rightarrow x_{0}$, implying $\beta=-\left(\omega+\frac{3}{2}\right)$. We have not been able to determine the parameters of $A$ and $\eta$ for general $\omega$.

If $\omega>1$, the situation is different. Now $M_{1+\omega}>M_{2}$ so that $M_{2}$ is bounded from below by [31], and the system suffers gelation, at least for $a>\frac{1}{4}$. In the steady state we write Eq. [40] as

$$
\begin{aligned}
0=a-M_{n} & +n M_{1+\omega} M_{n+\omega-1} \\
& +\frac{1}{2} \sum_{k=2}^{n-2}\left(\begin{array}{l}
n \\
k
\end{array}\right) M_{k+\omega} M_{n-k+\omega} .
\end{aligned}
$$

Since $M_{n+\omega-1}>M_{n}$ for $\omega>1$, the RHS must become strictly positive for some $n$. Hence the moment equation cannot be valid for all $n$, in the steady state, and we conclude that the distribution must have an algebraic tail. If we assume that $M_{2}$ is finite we again find that the coefficients of singular terms cannot be matched in the equation for the generating function; the only remaining possibility is gelation. Therefore we conclude that for $\omega$ $>1$ the steady state is gelling for all values of $a$, in other words $a_{0}(\omega)=0$ for $\omega>1$. However the case $\omega>1$ is unphysical $(4,7)$.

Summarizing, we have the following results for $K_{i j}=(i j)^{\omega}$ :

(i) If $\omega<\frac{1}{2}$, the system reaches a nongelling steady state for all values of the source rate $a$. The asymptotic behavior of the size distribution is dominated by exponential decrease.

(ii) For $\frac{1}{2}<\omega<1$ there is a number $a_{0}(\omega)$ such that the steady state is gelling for $a$ $>a_{0}(\omega)$ and nongelling for $a<a_{0}(\omega)$. For gelling steady states the size distribution has an algebraic tail $k^{-(3 / 2+\omega)}$ (Eq. [10]), for nongelling steady states dominated by an exponent of decreasing factor (Eq. [19] with $\beta$ $\left.=-\left(\omega+\frac{3}{2}\right)\right)$.

(iii) For $\omega=1$ the steady state is gelling for $a>\frac{1}{4}$ and has an algebraic tail $\sim k^{-5 / 2}$. For $a<\frac{1}{4}$ the system does not gel, but there is still an algebraic tail $\sim k^{\beta}$. The exponent $\beta=\left(-1+1 / M_{2}\right)$ is a known function of $a$.

(iv) For the unphysical case $\omega>1$ the steady state is always a gelling one, and has an algebraic tail $k^{-(3 / 2+\omega)}$.

It is in fact possible to calculate the steady state numerically in the following way. If the parameter $M_{\omega}$ which occurs in the equation were given, the steady-state equation could be solved recursively. So the parameter $M_{\omega}$ is varied and each time the size distribution is calculated, until the consistency requirement $M_{\omega}=\sum c_{k} k^{\omega}$ is met within a reasonable accuracy. It follows from the existence proof in the Appendix that the procedure converges to the unique steady state.

This way we have numerically confirmed the results (i) to (iv). For the parameter $M_{\omega}(a)$ upper and lower bounds can be found as 
follows: We take $0<\omega<1$. The equation for $M_{0}$ is given by

$$
a-M_{0}-M_{\omega}^{2} / 2=0
$$

and the fact that $M_{0}<M_{\omega}$ implies $M_{\omega}>(1$ $+2 a)^{1 / 2}-1$. On the other hand, from the steady-state equation, $M_{0}>c_{1}=a /\left(1+M_{\omega}\right)$ and this, combined with [43] leads to $M_{\omega}$ $<\frac{1}{2}\left|(1+8 a)^{1 / 2}-1\right|$, hence

$$
\begin{aligned}
(1+2 a)^{1 / 2}-1 & <M_{\omega} \\
& <\frac{1}{2}\left[(1+8 a)^{1 / 2}-1\right] .
\end{aligned}
$$

Since the bounds are asymptotically the same for large $a$, they also represent the large- $a$ behavior of $M_{\omega}(a)$. This implies that for large source rates the monomer is the dominant species in the system; which is to be expected physically.

\section{CONCLUSION}

We have found that in the presence of source and removal terms a unique steadystate solution exists to the coagulation Eqs. [1], when the kernel is a product of two factors $K_{i j}=s_{i} s_{j}$. The criterion for having a gelling steady state or not depends on the large- $k$ behavior of $s_{k}$ and on the relative strength of source and removal terms, expressed through the parameter $a^{\prime}=a / b^{2}$. For example, when $s_{k}=k^{\omega}$, gelling occurs for all values of $a^{\prime}$ when $\omega>1$, for $a^{\prime}>a_{0}^{\prime}(\omega)$ for $\frac{1}{2}<\omega<1$, and for no value of $a^{\prime}$ when $\omega$ $\leqslant \frac{1}{2}$. The conclusion for $\frac{1}{2}<\omega<1$ contradicts a conjecture in (2) that gelation always occurs for $\omega>\frac{1}{2}$. (This conjecture was based on the steady-state solution for a system without removal $(b=0)$, in which case the statement is true.) In all cases, except $\omega=1$, nongelling steady states correspond to exponentially dominated tails and gelling steady states to algebraic tails $k^{-(3 / 2+\omega)}$. For $\omega=1$, also the nongelling steady states have an algebraic tail $k^{\beta}$ where $\beta$ depends on the source rate $a$ as $\beta=-\left(1+2 /\left(1-(1-4 a)^{1 / 2}\right)\right)$. For $\omega=0$ the complete time-dependent solution could be given in terms of a generating function.

\section{APPENDIX}

Here we prove that for $K_{i j}=s_{i} s_{j}$ a unique steady-state solution of Eq. [2] exists for all values of $a$, irrespective of the functional form of $s_{k}$. The idea of the proof comes from an existence proof for a similar problem, given by Leyvraz (9). We define $a_{k}=s_{k} c_{k}$ and consider the recursion relation

$$
a_{k}=\frac{y \delta_{k 1}+\frac{1}{2} \sum_{i+j=k} a_{i} a_{j}}{s_{k}^{-1}+S},
$$

where $y$ and $S$ on which the $a_{k}$ depend are real and positive. A steady-state solution to [2] exists if we are able to construct $a$ value $S$ such that for $y=a$ the $a_{k}$ satisfies the consistency relation

$$
S=\sum_{k=1}^{\infty} a_{k}
$$

We shall, however, fix the value of $S$ first and show that there is a value $a$ of $y$ such that this condition is met. After that we show that $a$ is an invertible function of $S$, so that for every $a$ an $S$ can be found. Thus we fix $S$ and define, for $N=1,2,3 \cdots$, the numbers $b_{k}^{N}(y)$ by

$$
b_{k}^{N}(y)=\frac{y \delta_{k 1}+\frac{1}{2} \sum_{i+j=k} b_{i}^{N}(y) b_{j}^{N}(y)}{\theta(N-k) s_{k}^{-1}+S}
$$

in which $\theta(x)=0$ for $x<0$ and $\theta(x)=1$ for $x \geqslant 0$. The following properties hold:

$$
\begin{array}{ll}
b_{k}^{N}(y)=a_{k}(y) & k \leqslant N \\
b_{k}^{N}(y)>a_{k}(y) & k>N
\end{array}
$$

and from this

$$
b_{k}^{N+1}(y) \leqslant b_{k}^{N}(y)
$$

Furthermore, it follows from [A3] that the $b_{k}^{N}(y)$ are of the form

$$
b_{k}^{N}(y)=v_{k}^{N} y^{k}
$$

where $v_{k}$ does not depend on $y$ and, for $k$ $\leqslant N$, not on $N$. We define functions $F_{N}(y)$ and $P_{N}(y)$ as

$$
F_{N}(y)=\sum_{k=1}^{\infty} b_{k}^{N}(y)=\sum_{k=1}^{\infty} v_{k}^{N} y^{k}
$$




$$
P_{N}(y)=\sum_{k=1}^{N} b_{k}^{N}(y) s_{k}^{-1}=\sum_{k=1}^{N} a_{k}(y) s_{k}^{-1}
$$

$P_{N}(y)$ is a polynomial of degree $N$ in $y$. For $N=1$ the function $F_{1}(y)$ can be found from [A3] to be

$$
F_{1}(y)=S\left\{1-[1-2 y / S(S+1)]^{1 / 2}\right\}
$$

which has a radius of convergence $R_{1}=S(S$ $+1) / 2$. Because of [A5], for higher values of $N, F_{N}(y)$ must have a radius of convergence $R_{N} \geqslant R_{1}$. For all $y<R_{1}$ we derive from [A3], using the definition [A8]:

$$
F_{N}(y)=S-\left[Q_{M}(y)\right]^{1 / 2}
$$

with

$$
Q_{N}(y)=S^{2}+2\left[P_{N}(y)-y\right] . \quad \text { [A10] }
$$

The function $Q_{N}(y)$ is a polynomial in $y$ of degree $N$ which is a convex function on the real axis, since $Q_{N}^{\prime \prime}(y)=2 P_{N}^{\prime \prime}(y)>0$. Its zeroes correspond to singularities of $F_{N}(y)$. The power series of $F_{N}(y)$ has only positive coefficients so that the singularity nearest to the origin lies on the positive real axis, corresponding to a zero of $Q_{N}(y)$. Since $Q_{N}(y)$ is a convex polynomial it can have at most two real zeroes. If these two zeroes would coincide, $Q_{N+1}(y)$ would have no zero, since $Q_{M}(y)$ strictly increases with $N$, in contradiction with the fact that $F_{N}(y)$ has a singularity on the positive real axis. We conclude that $Q_{N}(y)$ must have two distinct zeroes $y_{N}$ and $y_{N}^{*}$ on the positive real axis and since $Q_{N+1}(y)$ $>Q_{N}(y)$ :

$$
y_{2}<y_{N}<y_{N+1}<y_{N+1}^{*}<y_{N}^{*}<y_{2}^{*},
$$

implying that the sequences $\left\{y_{N}\right\}$ and $\left\{y_{N}^{*}\right\}$ tend to the limits $y_{\infty}$ and $y_{\infty}^{*}$, respectively, with $y_{\infty} \leqslant y_{\infty}^{*}$. Since $P_{N}\left(y^{*}\right)<P_{N}\left(y_{N}\right)=-S^{2} /$ $2+y_{N} \rightarrow-S^{2} / 2+y_{\infty} P_{N}\left(y_{\infty}^{*}\right)$ also tends to a limit, which implies uniform convergence of the power series

$$
\lim _{N \rightarrow \infty} P_{N}(y)=\sum_{k=0}^{\infty} a_{k}(y) s_{k}^{-1}=P(y)
$$

for $y<y_{\infty}^{*}$. Using this result, the quantity

$$
\begin{aligned}
& \left|P\left(y_{\infty}\right)+S^{2} / 2-y_{\infty}\right| \\
& =\mid P\left(y_{\infty}\right)-P_{N}\left(y_{\infty}\right)+P_{N}\left(y_{\infty}\right)-P_{N}\left(y_{N}\right) \\
& \quad+P_{N}\left(y_{N}\right)+S^{2} / 2-y_{N}+y_{N}-y_{\infty} \mid \\
& =\mid\left[P\left(y_{\infty}\right)-P_{N}\left(y_{\infty}\right)\right]+\left[P_{N}\left(y_{\infty}\right)\right. \\
& \left.-P_{N}\left(y_{N}\right)\right]+\left[y_{N}-y_{\infty}\right] \mid
\end{aligned}
$$

can be made arbitrarily small. Hence,

$$
P\left(y_{\infty}\right)=y_{\infty}-S^{2} / 2 .
$$

For small enough $y\left(y<R_{1}\right)$, we may let $N \rightarrow \infty$ in [A10] to find

$$
\begin{aligned}
F(y) & =\lim _{N \rightarrow \infty} F_{N}(y) \\
& =S-\left[S^{2}+2(P(y)-y)\right]^{1 / 2} .
\end{aligned}
$$

Since $F(y)$ is an analytic function with only positive coefficients, its first singularity lies on the real axis. Because of [A 13] this singularity is in $y=y_{\infty}$ or further out on the real axis. ${ }^{1} F(y)$ is bounded on $\left(0, y_{\infty}\right)$ (from [A14]) and must be finite in $y_{\infty}$. So we can take the limit $y \rightarrow y_{\infty}$ in [A14] to find

$$
F\left(y_{\infty}\right)=\sum_{k=1}^{\infty} a_{k}(y)=S .
$$

So choosing $y=y_{\infty}=a$ in [A1] leads to a sequence $\left\{a_{k}\right\}$, satisfying the consistency relation [A2].

To show that conversely for a given value of $a(=y)$ a corresponding $S$ can be found we note that $y_{\infty}$ is a strictly increasing function of $S$ (see [A15]) that takes on all real values as $S \rightarrow \infty$, since $y_{\infty}>R_{1}=S(S+1)$ / 2. This function is continuous on account of [A13] and the fact that the series [A12] converges uniformly in $S$. Therefore $y_{\infty}$ is a bijective function of $S$, thus having an inverse.

This finally proves that for any value $a$ of $y$, a number $S$ exists such that both [A1] and [A2] are satisfied, constituting a steady-state solution to the coagulation equation in the presence of source and removal terms.

\footnotetext{
${ }^{1}$ When $y_{\infty}=y_{\infty}^{*}, F(y)$ is not singular there, since then the expression under the square root sign has a double zero in $y$.
}

Journal of Colloid and Interface Science, Vol. 105, No. 1, May 1985 


\section{ACKNOWLEDGMENTS}

Robert M. Ziff thanks the Shell Faculty Career Initiation Fund for support as a Shell Faculty Fellow. Eric Hendriks thanks the Chemical Engineering Department of the University of Michigan for hospitality. Both authors thank F. Leyvraz and P. V. Donger who carefully read the Appendix and clarified some obscure points in the proof.

\section{REFERENCES}

1. White, W. H., Colloid Interface Sci. 87, 204 (1982).

2. Crump, J. G., and Seinfeld, J. H., J. Colloid Interface Sci. 90, 469 (1982).
3. Lushnikov, A., and Piskunov, V. N., Dokl. Akad. Nauk SSSR 256, No. 51155 (1981).

4. Leyvraz, F., and Tschudi, H. R., J. Phys. A: Math. Gen. 14, 3389 (1981); 15, 1 (1981).

5. Ernst, M. H., Ziff, R. M., and Hendriks, E. M., J. Colloid Interface Sci. 97, 266 (1984).

6. Ziff, R. M., Ernst, M. H., and Hendriks, E. M., $J$. Phys. A: Math. Gen. 16, 2293 (1983).

7. Hendriks, E. M., Ernst, M. H., and Ziff, R. M., J. Stat. Phys. 3, 519 (1983).

8. Flory, P. J., "Principles of Polymer Chemistry." Cornell Univ. Press, Ithaca, N. Y., 1953.

9. Leyvraz, F., J. Phys. A: Math. Gen. 16, 2861 (1983).

10. Smoluchowski, M. v., Z. Phys. Chem. (in German) 92, 129 (1917). 